\title{
Compression of pressurised elastic pockets
}

\author{
Yann Bouremel ${ }^{\mathrm{a}, \mathrm{b}}$, Ian Eames ${ }^{\mathrm{b}, \mathrm{a}}$, Shivam Madaan ${ }^{\mathrm{c}}$, Steve Brocchinic,a, Peng \\ Tee Khaw ${ }^{\mathrm{a}}$ \\ ${ }^{a}$ National Institute for Health Research (NIHR) Biomedical Research Centre at \\ Moorfields Eye Hospital NHS Foundation Trust and UCL Institute of Ophthalmology, \\ London, UK \\ ${ }^{b}$ Department of Mechanical Engineering, University College London, London, UK \\ ${ }^{c} U C L$ School of Pharmacy, London, UK
}

\section{Abstract}

A circular silicone sheet is clamped at its edge and pressurised by the injection of a liquid beneath creating a pocket. We study experimentally the deformation and pressure increase caused by the compression of a pressurised pocket. Excellent agreement is found between experimental observations and numerical predictions based on an axisymmetric thin hyperelastic material; an approximate analytical model explains the link between changes in shape and the applied force.

Keywords: compression, deformation, analytical model, experiments, numerical model, elastic pocket, soft button

\section{Introduction}

Planar elastic pockets are formed by introducing a fluid at pressure beneath a deformable sheet. They are used in hydraulic systems, such as JPmate Air Jack from Hornchic, to provide a vertical force to lift heavy objects and are quite versatile because they can be made thin and inserted into narrow spaces. Pressurised elastic pockets have been used as a sensing device 
since the 1880's with the invention of diaphragm-type optical indicator (eg the Clarke \& Low, the Perry and the Bedell indicators (Walter, 2011) among others) made of thin sheet steel to monitor the pressure level of steam engine; the deformation of a hemispherical elastic diaphragm displaced mirrors that reflected a light beam on to a photographic plate (Bedell, 1897). The complexity of this problem arises because the initial and deformed shape of the pocket are determined by the initial level of pressurisation, material properties, planform area of the pocket and the applied force.

The deformation of closed spherical or elliptical shells by an applied force has been extensively studied experimentally and theoretically driven by attempts to infer the mechanical properties of biological components, such as fish eggs, sea urchins (Vlès, 1926; Cole, 1932), microcapsules (Liu et al., 1996; Carin et al., 2003) and cells (Smith et al., 1998) with earlier experiments dating back from 1891 (Pfeffer, 1891). Cells in their fluid environment are under stress that may lead to damage when under excessive compression in typical bioprocesses for example Arnaud et al. (1993) and Ho et al. (1995), or more recently, the studies of Douaire et al. (2011) on the phenotypic modification of bacteria in a Couette bioreactor. The cell response can be modelled as a closed axisymmetric elastic shell (Smith et al., 1998). In all cases, the problem of damage is a general one arising from cells interacting with their fluid environment (Zhang and Thomas, 1993) as formalised in the wall-strength model for microbial cell disruption (Middelberg et al., 1992a,b). On a larger scale, elliptical shells can also be used as air-inflated building structures (Bolonkin and Cathcart, 2007; Bolonkin et al., 2011; Kozicki and Kozicka, 2011) which are dome-shaped as they provide the greater 
surface area under the structure for a minimum amount of material. The applications of planar elastic pockets are therefore very diverse.

The current paper aims at designing soft buttons. Soft buttons are able to provide different pressure responses depending on their deformations. They give an alternative to the classic "on/off" function of classic buttons.

The soft button is modelled as a pocket with a circular contact perimeter and clamped. The goal of the paper is to establish the relation between the button deformation when squashed and its pressure response. Figure 1(a) shows a circular clamped sheet of radius $R$ and thickness $T$ that is pressurised by introducing a fluid beneath the sheet (to give the pressurised state in figure 1(b)). A force is applied to the pressurised pocket to give a squashed state (figure $1(\mathrm{c})$ ). The elastic sheet is thin (i.e. $T / R \ll 1$ ) and axisymmetric, and so can be modelled as a thin hyper-elastic sheet using the framework of Feng and Yang (1973) as well as Yang and Feng (1970) to a lesser extent. The current study examines the correlation between the increase of pressure and area of contact when pockets are under compression.

\section{Mathematical model for the pocket}

\subsection{Formulation}

The essential features of the thin sheet model are based on the work of Yang and Feng (1970) and Feng and Yang (1973). The incompressible elastic material is modelled using a strain-energy density function $W$ that follows Mooney-Rivlin (Mooney, 1940):

$$
W\left(I_{1}, I_{2}\right)=C_{1}\left(\left(I_{1}-3\right)+\alpha\left(I_{2}-3\right)\right),
$$


with $C_{1}, \alpha=C_{2} / C_{1}$ being the Mooney-Rivlin constants and $I_{1}, I_{2}$, the strain invariants. The Young's modulus, $E$, in the limit of uniaxial tension is related to the Mooney-Rivlin constants $C_{1}$ and $\alpha$ through $E=6(1+\alpha) C_{1}$. In this model, the stretch factors and membrane stress resultants are expressed and solved in the Lagrangian coordinate $r$ of the underformed initial circular flat sheet and then converted to Eulerian coordinates $(\rho, \eta)$ following the work of Yang and Feng (1970). In Figure 1, the meridian and hoop stretch ratio denoted respectively $\lambda_{1}$ and $\lambda_{2}$ are

$$
\begin{aligned}
\lambda_{1}=\frac{\mathrm{d} s}{\mathrm{~d} r}= & \left(\left(\frac{\mathrm{d} \rho}{\mathrm{d} r}\right)^{2}+\left(\frac{\mathrm{d} \eta}{\mathrm{d} r}\right)^{2}\right)^{1 / 2}, \\
& \lambda_{2}=\frac{\rho}{r}
\end{aligned}
$$

where $r$ and $\rho$ are the radial distances of the undeformed and deformed elements of the sheet respectively and $s$ and $\eta$ its pursed meridian arc length and height.

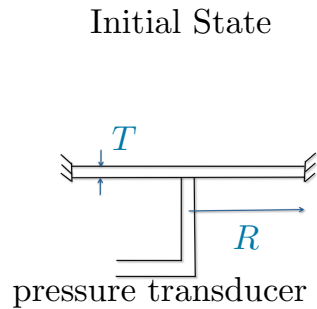

(a)

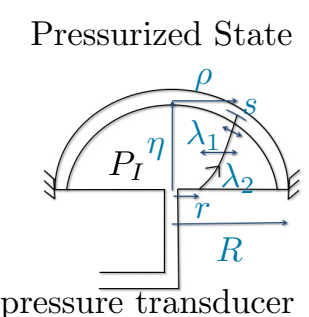

(b)

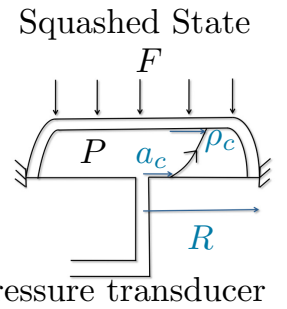

(c)

Figure 1: (a): Schematic of a pocket of thickness, $T$, in the initial state then (b) pursed with a uniform pressure $P_{I}$ which is compressed in (c) with a hydrostatic force applied uniformly on the top of pocket that results to a pressure inside the pocket equal to $P$. The Lagrangian coordinates $(r, 0)$ map onto the Eulerian coordinates $(\rho, \eta)$.

The stretch factor in the through-surface direction, $\lambda_{3}$, is determined by 
the incompressibility constraint

$$
\lambda_{1} \lambda_{2} \lambda_{3}=1
$$

The local equilibrium of forces on a segment of the elastic sheet in the normal and tangential directions are (Yang and Feng, 1970)

$$
\begin{gathered}
\kappa_{1} T_{1}+\kappa_{2} T_{2}=P, \\
\frac{\partial T_{1}}{\partial \rho}+\frac{1}{\rho}\left(T_{1}-T_{2}\right)=0,
\end{gathered}
$$

with $\kappa_{1}, \kappa_{2}$ being the principal curvatures and $T_{1}, T_{2}$, the membrane stress resultants in the meridional and circumferential directions and $P$, the pressure in the pocket. The curvatures in the meridian and circumferential directions are given by

$$
\kappa_{1}=\frac{-\frac{\mathrm{d}^{2} \eta}{\mathrm{d} \rho^{2}}}{\left(1+\left(\frac{\mathrm{d} \eta}{\mathrm{d} \rho}\right)^{2}\right)^{3 / 2}}, \quad \kappa_{2}=-\frac{\frac{\mathrm{d} \eta}{\mathrm{d} \rho}}{\rho\left(1+\left(\frac{\mathrm{d} \eta}{\mathrm{d} \rho}\right)^{2}\right)^{1 / 2}}
$$

The components of membrane stress resultants that are consistent with (1) and (4) are

$T_{1}=2 T C_{1}\left(\frac{\lambda_{1}}{\lambda_{2}}-\frac{1}{\lambda_{1}^{3} \lambda_{2}^{3}}\right)\left(1+\alpha \lambda_{2}^{2}\right), \quad T_{2}=2 T C_{1}\left(\frac{\lambda_{2}}{\lambda_{1}}-\frac{1}{\lambda_{1}^{3} \lambda_{2}^{3}}\right)\left(1+\alpha \lambda_{1}^{2}\right)$.

When the pocket is pressurised, a new variable $w$ is introduced and defined as $w=\frac{\mathrm{d}\left(\lambda_{2} \widetilde{r}\right)}{\mathrm{d} \widetilde{r}}$, and the components of the membrane stress resultants are described by the system of equations $\left(\lambda_{1}, \lambda_{2}, w\right)$ whose gradients are linked 
through (Yang and Feng, 1970)

$$
\begin{aligned}
\frac{\mathrm{d} \lambda_{1}}{\mathrm{~d} \widetilde{r}}= & \frac{1}{\widetilde{r}}\left\{\frac{w}{\lambda_{2}}\left[\frac{\lambda_{2}}{\lambda_{1}}-\frac{\lambda_{1}}{\lambda_{2}}-\alpha\left(\frac{1}{\lambda_{1} \lambda_{2}^{3}}-\frac{1}{\lambda_{1}^{3} \lambda_{2}}\right)\right]\right. \\
& \left.-\left(w-\lambda_{2}\right)\left[\left(-\frac{\lambda_{1}}{\lambda_{2}^{2}}+\frac{3}{\lambda_{1}^{3} \lambda_{2}^{4}}\right)\left(1+\alpha \lambda_{2}^{2}\right)+2 \alpha \lambda_{2}\left(\frac{\lambda_{1}}{\lambda_{2}}-\frac{1}{\lambda_{1}^{3} \lambda_{2}^{3}}\right)\right]\right\} \\
& \frac{1}{\left(\frac{1}{\lambda_{2}}+\frac{3}{\lambda_{1}^{4} \lambda_{2}^{3}}\right)\left(1+\alpha \lambda_{2}^{2}\right)}, \\
\frac{\mathrm{d} w}{\mathrm{~d} \widetilde{r}}= & \frac{1}{\widetilde{r}} \frac{\lambda_{2}}{\mathrm{~d} \widetilde{r}}=\frac{w-\lambda_{2}}{\widetilde{r}}, \\
& {\left[\frac{1}{\lambda_{2}}-\frac{1}{\lambda_{1}^{3} \lambda_{2}^{3}}\right)\left(1+\alpha \lambda_{2}^{2}\right) } \\
& +\frac{w}{\lambda_{1} \lambda_{2}} \frac{\mathrm{d} \lambda_{1}}{\mathrm{~d} \widetilde{r}},
\end{aligned}
$$

with

$$
\hat{P}=\frac{P R}{E T}, \quad \tilde{r}=\frac{r}{R} .
$$

When the pocket is squashed, the above equations applied to the portion of the sheet that is not in contact with the applied force, i.e. $\tilde{a}_{c} \leq \tilde{r} \leq 1$ (when $\tilde{a}_{c}=a_{c} / R$ ). In the region of the membrane in contact with the plate $\left(\tilde{r} \leq \tilde{a}_{c}<1\right)$, the sheet is flat so that $\mathrm{d} \eta / \mathrm{d} r=0$. This corresponds to a distance $0 \leq \tilde{r} \leq \tilde{a}_{c}$. In this region, (7) and (8) still hold true while (9) is replaced by:

$$
\frac{\mathrm{d} w}{\mathrm{~d} \tilde{r}}=\frac{\mathrm{d} \lambda_{1}}{\mathrm{~d} \tilde{r}} .
$$

The height $\eta$ of the pressurised pocket can be rewritten following (2):

$$
\frac{\eta}{R}=\int_{\tilde{r}}^{1}\left(\lambda_{1}^{2}-\left(\frac{\mathrm{d} \rho}{\mathrm{d} \tilde{r}}\right)^{2}\right)^{1 / 2} \mathrm{~d} \tilde{r} .
$$


The volume of fluid contained beneath the elastic sheet is

$$
V=\int_{0}^{\eta_{\max }} \pi \rho^{2} \mathrm{~d} \eta
$$

\subsection{Numerical solution procedure}

Two steps are required to calculate the response of a pocket to an applied force.

- Step 1: The applied pressure $P_{I}$ is defined and the shape of the deformed circular disc is determined with $\lambda_{1}=\lambda_{2}=\lambda_{0}=w$ at $\tilde{r}=0$. A search algorithm is then run to determine the value of $\lambda_{0}$ using a second boundary condition that differs from the original problem solved by Feng and Yang (1973) which satisfies $\lambda_{2}(\tilde{r}=1)=1$ using equations (7), (8) and (9) with a bisection method. This condition shows that the radial distance of the boundary of the pocket is fixed and will not change when the pocket is under compression. Once the shape is determined, the volume under the pocket, $V$, is calculated using (12) and (13) and recorded.

- Step 2: The pocket is compressed with a pressure $P$ and (7), (8) and (11) are solved using the same technique and similar boundary conditions as Step 1. The radius $\tilde{a}_{c}$ is unknown and this step is solved interactively to determine the value of $a_{c}$ such that the volume is the same as Step 1. The search solution in both steps were solved in Matlab 2012 with a tolerance of $10^{-4 \%}$ usually obtained in less than 20 iterations. The step when searching the contact radius can be as small as $0.5 \%$ of the pocket radius. 


\section{Analytical model}

To understand the physics of the compression process, we attempt to develop a simple model; the case of pressurised pocket and squashed pocket are treated separately because there are significant differences. For low applied pressures $P_{I}$ when the stretch factor $\left|\lambda_{1}-1\right| \ll 1$, we can approximate the stretch factors as

$$
\lambda_{1} \cong \lambda_{0}, \quad \lambda_{2} \cong \lambda_{0}-\left(\lambda_{0}-1\right) \tilde{r}^{2}
$$

to ensure the boundary conditions $\lambda_{1}=\lambda_{2}=\lambda_{0}$ at $\tilde{r}=0$ with $\lambda_{0}$ being a constant and $\lambda_{2}=1$ at $\tilde{r}=1$. With this prescribed from, the shape of the pocket can be estimated from (12),

$$
\frac{\eta}{R} \cong\left(\frac{3}{2}\left(\lambda_{0}-1\right)\right)^{\frac{1}{2}}\left(1-\tilde{r}^{2}\right) .
$$

The pocket height and volume are

$$
\begin{aligned}
& \frac{H_{0}}{R} \approx\left(\frac{3}{2}\left(\lambda_{0}-1\right)\right)^{\frac{1}{2}}, \\
& \frac{V}{R^{3}} \approx \frac{\pi}{4}\left(6\left(\lambda_{0}-1\right)\right)^{\frac{1}{2}} .
\end{aligned}
$$

At the centre of the pocket, $T_{1}=T_{2}$ and $\kappa_{1}=\kappa_{2}$, where

$$
\begin{aligned}
& T_{1} \cong 12 C_{1} T\left(\lambda_{0}-1\right) \\
& \kappa_{1} R \cong\left(6\left(\lambda_{0}-1\right)\right)^{\frac{1}{2}}
\end{aligned}
$$

From (4), the pressure can be determined from the force balance at $\tilde{r}=0$ giving

$$
P_{I} \cong 24 C_{1} \frac{T}{R} \sqrt{6}\left(\lambda_{0}-1\right)^{\frac{3}{2}}
$$


Full numerical calculations confirm that the above model is good when $\lambda_{0} \leq$ 1.1 (Bouremel et al., 2017), beyond which the analysis breaks down since the increase of $\lambda_{1}$ with $\tilde{r}$ needs to be taken into account. When the pocket is compressed, within the contact region $\lambda_{1}=\lambda_{2}=\lambda_{c}$ are constants, while outside the contact-region, we approximate the stretch factors as

$$
\lambda_{1} \cong \lambda_{c}, \quad \lambda_{2} \cong \lambda_{c}-\left(\lambda_{c}-1\right) \frac{\tilde{r}^{2}-\tilde{a}_{c}^{2}}{1-\tilde{a}_{c}^{2}},
$$

to ensure that the boundary conditions at $\tilde{r}=1$ are satisfied. The normalised deflection, $\frac{\eta}{R}$, can be rewritten as:

$$
\frac{\eta}{R}=\left(\lambda_{c}-1\right)^{\frac{1}{2}} G\left(\tilde{r}, \tilde{a}_{c}\right)
$$

where

$$
G\left(\tilde{r}, \tilde{a}_{c}\right)= \begin{cases}\frac{\sqrt{2}}{\left(1-\tilde{a}_{c}^{2}\right.} \int_{\tilde{a}_{c}^{\frac{1}{2}}}^{1}\left(3 \tilde{r}^{2}-\tilde{a}_{c}^{2}\right)^{\frac{1}{2}} \mathrm{~d} \tilde{r}, & \tilde{r} \leq \tilde{a}_{c}, \\ \frac{\sqrt{2}}{\left(1-\tilde{a}_{c}^{2}\right)^{\frac{1}{2}}} \int_{\tilde{r}}^{1}\left(3 \tilde{r}^{2}-\tilde{a}_{c}^{2}\right)^{\frac{1}{2}} \mathrm{~d} \tilde{r}, & \tilde{r} \geq \tilde{a}_{c} .\end{cases}
$$

The pocket height is

$$
\frac{H}{H_{0}}=\sqrt{\frac{2}{3}\left(\frac{\lambda_{c}-1}{\lambda_{0}-1}\right)} G\left(0, \tilde{a}_{c}\right),
$$

where $H_{0}$ corresponds to (16a): pocket volume must remain unchanged when squashed so that

$$
V=\left(\lambda_{c}-1\right)^{\frac{1}{2}} 2 \pi \int_{0}^{1} \tilde{r} G\left(\tilde{r}, \tilde{a}_{c}\right) \mathrm{d} \tilde{r}=\left(\lambda_{0}-1\right)^{\frac{1}{2}} \frac{\sqrt{6} \pi}{4} .
$$

The volume constraint determines the relationship between $\lambda_{c}$ and $\tilde{a}_{c}$; since the model is appropriate for small values of $\lambda_{c}$, then

$$
\frac{\lambda_{c}-1}{\lambda_{0}-1}=\left(\frac{\sqrt{6}}{8 \int_{0}^{1} \tilde{r} G\left(\tilde{r}, \tilde{a}_{c}\right) \mathrm{d} \tilde{r}}\right)^{2} .
$$


When the pocket is not compressed, i.e. $\tilde{a}_{c}=0$, we recover $\lambda_{c}=\lambda_{0}$. We link the pressure of the squashed pocket to the forces on the sheet at the contact region $\tilde{r}=\tilde{a}_{c}$ where

$$
T_{1}=T_{2} \cong 12 C_{1} T\left(\lambda_{c}-1\right)
$$

The radii of curvature of the pocket at $\tilde{r}=\tilde{a}_{c}$ are now different with the meridional curvature being much larger than the circumferential component, giving

$$
\begin{aligned}
& \kappa_{1} R \cong 3\left(\frac{\lambda_{c}-1}{1-\tilde{a}_{c}^{2}}\right)^{\frac{1}{2}} \\
& \kappa_{2} R \cong 2\left(\frac{\lambda_{c}-1}{1-\tilde{a}_{c}^{2}}\right)^{\frac{1}{2}} .
\end{aligned}
$$

This approximation is singular in the sense that in the limit of $\tilde{a}_{c} \rightarrow 0$, the radii of curvature does not tend to $(17 \mathrm{~b})$.

\section{Experimental}

\subsection{Set-up and Methodology}

The experimental set up consisted of an elastic silicone sheet sandwiched between a clear base acrylic plate and a steel upper plate; a circular hole was cut into the top plate so that by introducing water beneath the sheet enables it to be pressurised to an initial pressure $P_{I}$.

The source to the circular disc was connected via a three way valve to a vertical tube that enabled the initial pressure of the pocket to be set using a hydrostatic head and then the connection was sealed and connected to a calibrated pressure transducer. The water head varied from 20 to $50 \mathrm{~cm}$ significantly larger than the height of the pocket, so that the pressure variation 
with the height in the pocket is negligible. After applying a force, $F$, the pressure in the chamber increased to $P$. The force was applied uniformly over the top of the chamber by using custom-made cylinder weights. The contact between the applied force $F$ and the elastic membrane generates an area $A_{c}$ and is related through $A_{c}=F / P$. The height of the squashed pocket was measured using a photographic technique.

\subsection{Material characterisation}

Silicone sheets with different thicknesses ranging from $0.25 \mathrm{~mm}$ to $3.2 \mathrm{~mm}$ were sourced from Silex Ltd (Broxhead Trading Estate, Lindford, Bordon, Hampshire, GU35 OJX, UK). The material properties were characterised using an electromagnetic linear actuator (Bose Electroforce 3220 Series III to determine $C_{1}, C_{2}$ and $E$ ). The experimental procedure is described in Bouremel et al. (2017) and the samples used are different. The MooneyRivlin parameters of the silicone sheets are listed in Table 1.

\begin{tabular}{c|cccccc}
$\begin{array}{c}\text { silicone } \\
\text { sheet }\end{array}$ & $\begin{array}{c}\text { Thickness } \\
T\end{array}$ & $C_{1}$ & $C_{2}$ & $\begin{array}{c}\text { Young Modulus } \\
E\end{array}$ & Symbol & $P_{I}$ \\
sample & $(\mathrm{mm})$ & $(\mathrm{kPa})$ & $(\mathrm{kPa})$ & $(\mathrm{MPa})$ & & $(\mathrm{kPa})$ \\
\hline 1 & 0.25 & 160 & 47 & 1.241 & $\mathbf{\nabla}$ & $2-5$ \\
2 & 0.5 & 160 & 47 & 1.241 & $\boldsymbol{\nabla}$ & $2-5$ \\
3 & 0.8 & 160 & 47 & 1.241 & $\mathbf{4}$ & $2-5$ \\
4 & 1.6 & 160 & 47 & 1.241 & $\mathbf{\square}$ & $2-5$
\end{tabular}

Table 1: Table summarising the silicone samples used in the experimental study. The sheet thickness $(T)$, Mooney-Rivlin parameters $C_{1}$ and $C_{2}$, Young Modulus $(E)$, and symbols are listed. 


\section{Results}

The elastic pockets are filled with water and have a dome shape as shown in Figure 2 (a) where sheet sample 1 is clamped along a $2 \mathrm{~cm}$ radius circle and pressurised at $\hat{P}_{I}=0.31$ with $\hat{P}$ defined in (10). The numerical green line obtained with the method explained in Section 2.2 follows the shape contours of the post-processed photograph of our pocket. When the pockets are compressed, they deform with an increase in the hydrostatic pressure. They are flatten on the top of their surfaces while bulging along the side to retain their volume of water as shown in Figure 2 (b) with the numerical line obtained in green for $F / P_{I} \pi R^{2}=0.19$.

The shape deformation can be studied by looking at the evolution of the contact area, $A_{c}$, and the deflection of the pocket at $\tilde{r}=0, H$. Figure 3 (a) shows that the contact area (flat surface on the top) compressing the pocket varies non-linearly with the compressive force $F=P_{I} \pi R^{2}$. The numerical line plotted in magenta is obtained from a set of simulations for different pocket radii, $R$ from $0.01 \mathrm{~m}$ to $0.02 \mathrm{~m}$, thickness, $T$ from $2.5 \times 10^{-4} \mathrm{~m}$ to $2 \times 10^{-3} \mathrm{~m}$, and material characteristic, $\alpha$ from 0.3 to 1.2. At large applied forces, the contact area tends to a constant. The comparison between experimental points and the numerical results shows a very good agreement across the applied force range. The analytical model developed in section (3) is shown with a black line. The agreement is good for low compressive forces $\left(F / P_{I} \pi R^{2}<0.1\right)$. However, as the compressive force increases, the increase of meridional stretch factor becomes important, it leads to an under prediction of $A_{c}$. Figure $3(\mathrm{~b})$ shows the decrease of the purse height, $H$, normalised by the equilibrium height, $H_{0}$, as the compressive force is increased. This 


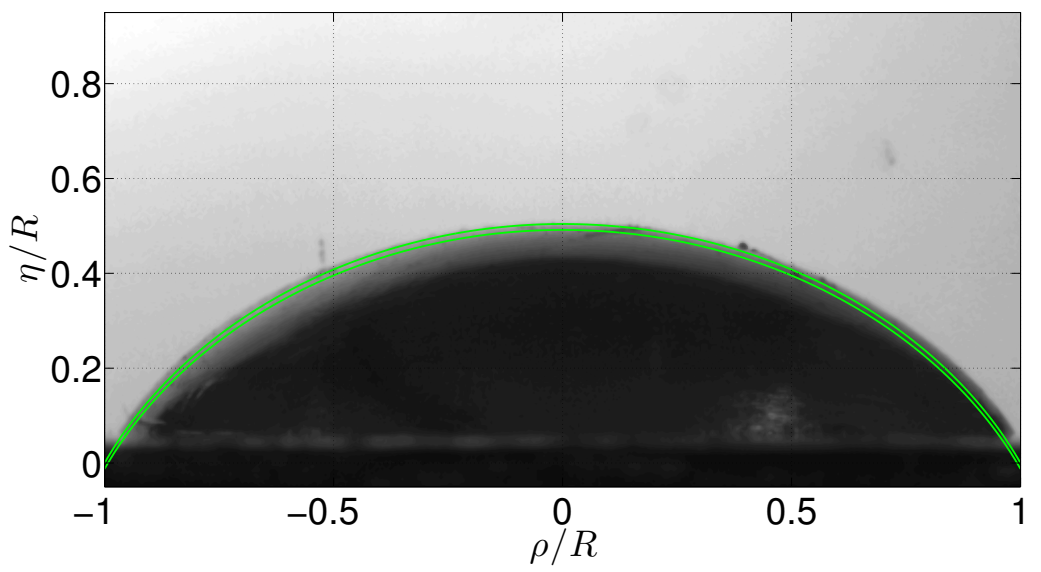

(a)

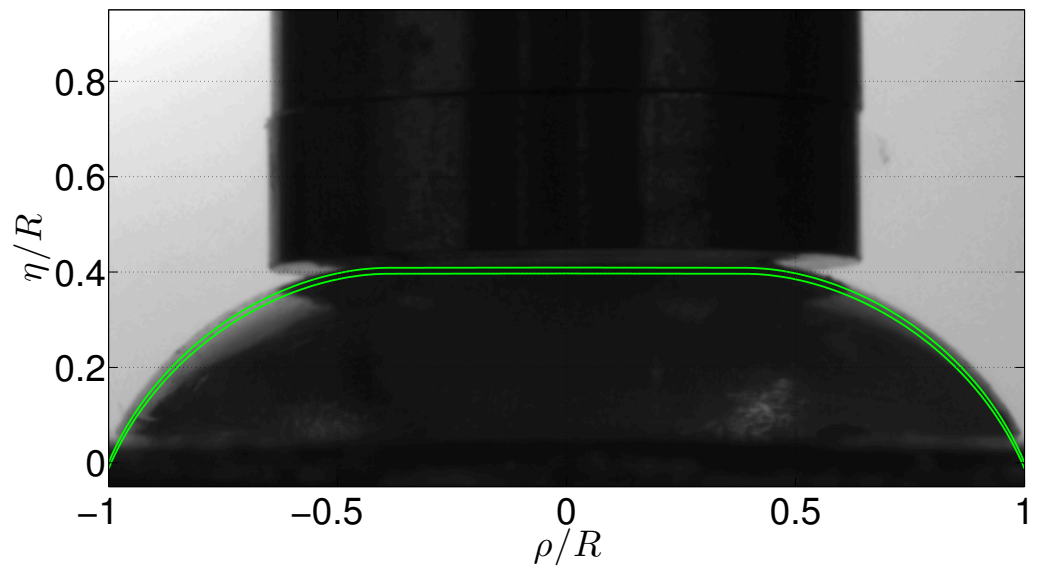

(b)

Figure 2: Post-processed images using sheet sample 1 pursed at a pressure $\hat{P}_{I}=0.31$ with the numerical profiles superimposed and plotted in green for (a) $F / P_{I} \pi R^{2}=0$ and (b) $F / P_{I} \pi R^{2}=0.19$. 
decrease of $H$ mirrors the increase of $A_{c}$ noted in Figure 3 (a) to maintain the pocket volume constant throughout the compression. The pocket height is quite well captured over a wide range of $F$ by the numerical model plotted with a magenta line and the analytical model shown with a black line. 


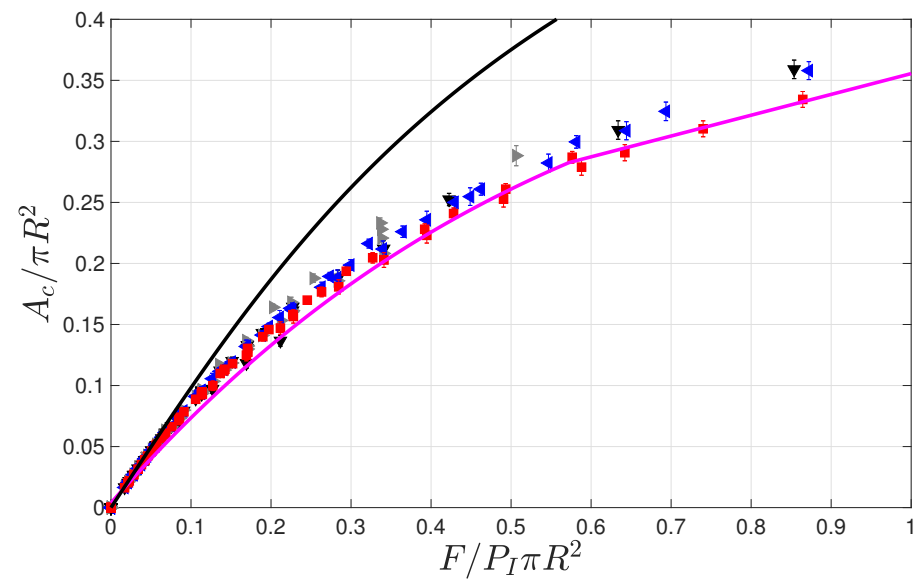

(a)

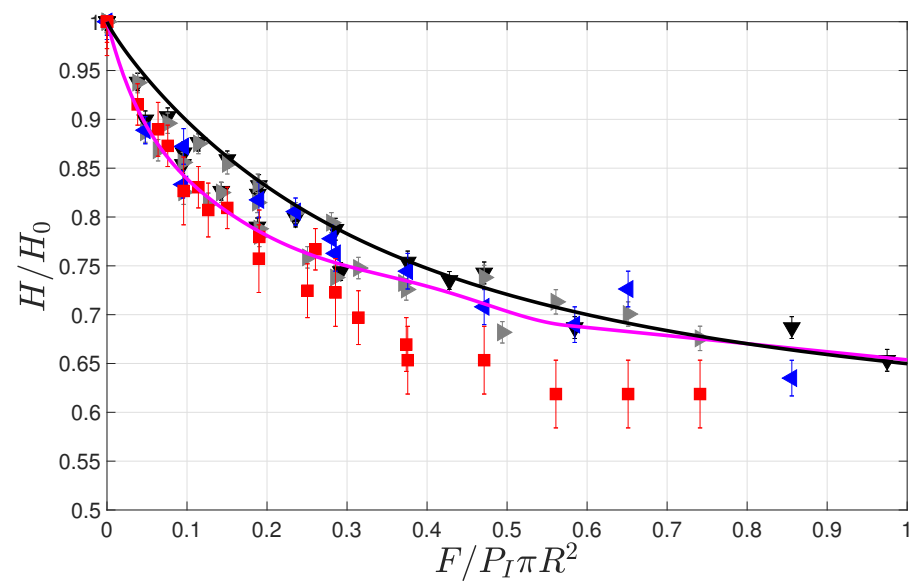

(b)

Figure 3: Variation of the contact area fraction $A_{c} / \pi R^{2}$ (a) and normalised deflection height $H / H_{0}$ (b) obtained at $\tilde{r}=0$ of pockets made with the different samples listed in Table 1 under increasing compressive forces $F / P_{I} \pi R^{2}$. The numerical line in magenta is obtained from a range of simulations with pockets of different size and thickness and made of material with different parameters, $\alpha$. The black line is obtained analytically using (20) - (23). 
Figure 4 shows the maximum deflection non-dimensionalised by the radius, $H / R$, of pockets of different samples listed in Table 1 under compression when initially pursed with the same pressure ( $\hat{P}_{I}$ from 0.05 to 0.32$)$ to complement the results of Figure 3 (b). The non-dimensional compression $P / P_{I}$ is related to the compressive forces $F / P_{I} \pi R^{2}$ defined in Figures 3 (a) and (b) through the contact area fraction, $A_{c} / \pi R^{2}: F / P_{I} \pi R^{2}=P / P_{I} \times A_{c} / \pi R^{2}$. An excellent agreement between the simulations and the experiments with the general conclusion that pockets made of thinner material are associated to higher deflections.

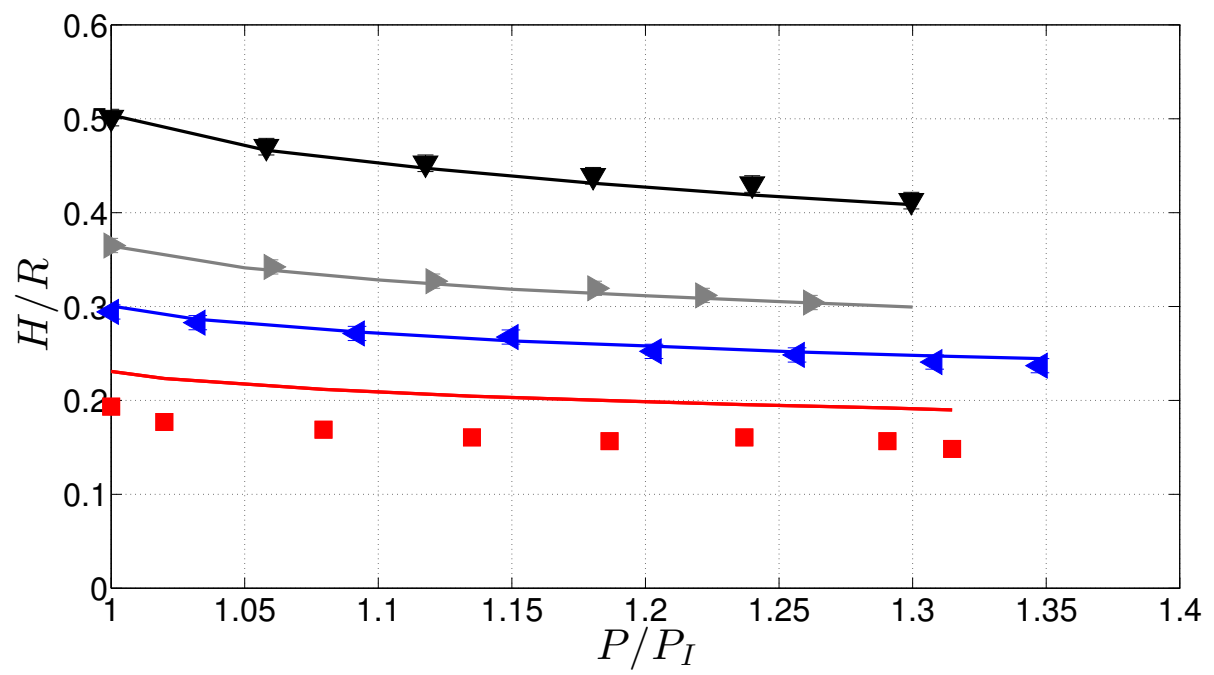

(a)

Figure 4: Variation of the maximum deflection height $H / R$ obtained at $\tilde{r}=0$ of pockets made with the different samples listed in Table 1 under compression $P / P_{I}$ for the same initial pressure and $\hat{P}_{I}$ ranging from 0.05 to 0.32 . The equivalent numerical line is plotted with a line of the same colour.

Figure 5 shows the relative hydrostatic pressure increase $P / P_{I}$ when com- 
pressed with the relative compressive force $F / P_{I} \pi R^{2}$. Similarly to Figure 3, the numerical line plotted in magenta is obtained from a set of simulations for different pocket radii, $R$ from $0.01 \mathrm{~m}$ to $0.02 \mathrm{~m}$, thickness, $T$ from 2.5 $\times 10^{-4} \mathrm{~m}$ to $2 \times 10^{-3} \mathrm{~m}$, and material characteristic, $\alpha$ from 0.3 to 1.2 . This shows an approximately linear relationship between the pressure and the compression force.

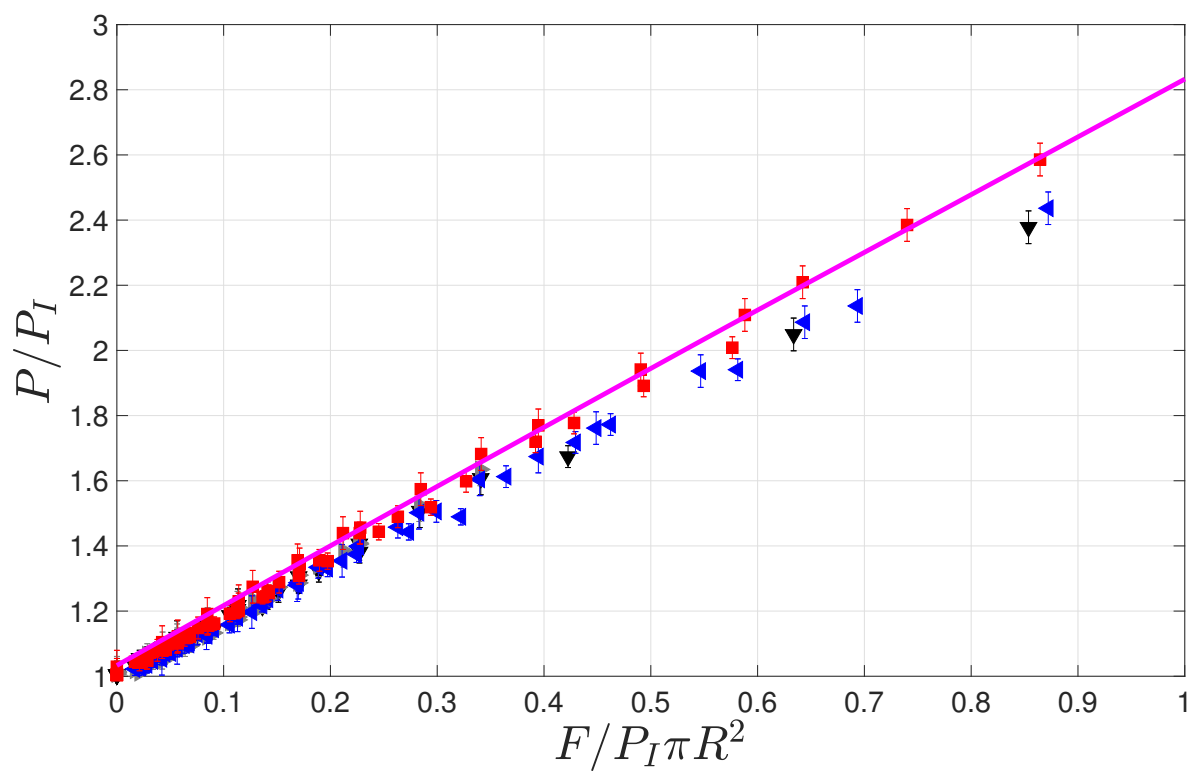

Figure 5: Variation of the internal pressure $P / P_{I}$ of pockets made with the different samples listed in Table 1 when under increasing compressive forces $F / P_{I} \pi R^{2}$. The numerical line in magenta is obtained from a range of simulations, with pockets of different size and thickness and made of material with different parameters, $\alpha$.

\section{Designing a soft button}

The experimental study and theoretical computations enable the undeformed and deformed states to be related to $H_{0}, E, T, R, F$ and $P_{I}$. We 
now apply these results for designing a soft button. The two major constraints on button design are (a) geometrical and (b) ergonomical, which are summarised in Table 2.

The geometrical constraints in the initial undeformed state are $R$ and $H_{0}$. U.S Department of Defense (1999) released a standardised human engineering criteria design report for push buttons among other equipments. This report mentions that push buttons should have a minimum diameter of 10 $\mathrm{mm}$ when pushed using fingertips while a minimum of $19 \mathrm{~mm}$ when using the thumb showing the natural larger area accommodated by different fingers. In this example, we are looking at buttons pressed by a single finger with $R \approx 20 \mathrm{~mm}$ and $H_{0} \approx 10 \mathrm{~mm}$.

The ergonomic constraints are softness and tactility. For softness, we require compliant smooth material and choose silicone which is characterised by a Young's modulus of $E \approx 10^{6} \mathrm{~Pa}$. For the button to be tactile, it must deform due to an applied force, $F$ and the typical deformation must be a reasonable fraction of the button height, i.e $H / H_{0} \approx 0.8$. From Figure 3 (b), it can be calculated that the tactile nature of the button sets the initial pressure through $P_{I} \approx 5.7 F / \pi R^{2}$ with $H / H_{0} \approx 0.8$. A typical value of $F$, for a finger, is approximately $0.67 \mathrm{~N}$. Finally, the sheet thickness, $T$, can be determined from the initial button height $H_{0}$, radius $R$, pressure $P_{I}$ and $E$ through $T=0.216 P_{I} R^{4} / E H_{0}^{3}$ (Bouremel et al., 2017). 


\begin{tabular}{|l|c|c|}
\hline Constraints & Parameters & Values \\
\hline Size of button & $R$ & $\approx 20 \mathrm{~mm}$ \\
Height of button & $H_{0}$ & $\approx 10 \mathrm{~mm}$ \\
Soft e.g. silicone & $E$ & $\approx 10^{5}-10^{6} \mathrm{~Pa}$ \\
Tactile & $P_{I}$ & $5.7 F / \pi R^{2}\left(\right.$ so $\left.H / H_{0} \approx 0.8\right)$ \\
Thickness of button & $T$ & $0.216 P_{I} R^{4} / E H_{0}^{3}$ \\
Maximum force & $F$ & $\approx 0.67 N$ \\
\hline
\end{tabular}

Table 2: Table summarising the constraints on the design of a soft button.

\section{Conclusions}

We have studied circular pockets experimentally and numerically, by drawing on axisymmetric models of hyper-elastic sheets, and shown how simplified models largely explain their function for small applied forces. For low initial loads, the application of a force reduces the stretch factor in the centre of the pocket, but increases the meridional stretch factors at the edge. The volume constraint of the pocket sets the relationship between the pressurised and squashed states. The analysis shows that the applied force can be inferred from the pressure increase meaning that the force range can be accurately determined. By correlating the shape, the pressure and the applied force, the evolution of compressed systems that can be simplified as elastic pocket such as microcapsules can be determined in the limit of small applied forces in constrained spaces. Finally, the theory can be applied to design soft tactile buttons to provide different pressure responses depending on their deformations. 
Acknowledgements. The authors acknowledge the support of the UK National Institute for Health Research Biomedical Research Centre at Moorfields Eye Hospital and the UCL Institute of Ophthalmology, the Helen Hamlyn Trust in memory of Paul Hamlyn, and Moorfields Eye Charity. 
Arnaud, J. P., Lacroix, C., Foussereau, C., Choplin, L., 1993. Shear stress effects on growth and activity of Lactobacillus delbrueckii subsp. bulgaricus. Journal of Biotechnology 29, 157-175.

Bedell, C. H., 1897. Steam engine indicator. Patent No. 575,202.

Bolonkin, A., Neumann, S., Friedlander, J., 2011. Transparent Inflatable Column Film Dome for Nuclear Stations, Stadiums, and Cities. Science and Technology of Nuclear Installations 2011, 1-13.

Bolonkin, A. A., Cathcart, R. B., 2007. Inflatable 'Evergreen' dome settlements for Earth's Polar Regions. Clean Techn Environ Policy 9, 125-132.

Bouremel, Y., Madaan, S., Lee, R., Eames, I., Wojcik, A., Khaw, P. T., 2017. Pursing of planar elastic pockets. Journal of Fluids and Structures $70,261-275$.

Carin, M., Barthès-Biesel, D., Edwards-Lévy, F., Postel, C., Cristina Andrei, D., 2003. Compression of biocompatible liquid-filled HSA-alginate capsules: determination of the membrane mechanical properties. Biotechnology and bioengineering 82 (2), 207-212.

Cole, K. S., 1932. Surface forces of the arbacia egg. Journal of cellular and comparative physiology 1 (1), 1-9.

Douaire, M., Mercade, M., Morchain, J., Loubière, P., 2011. A Unique Phenotypic Modification of Lactococcus lactis Cultivated in a Couette Bioreactor. Biotechnology and Bioengineering 108 (3), 559-571. 
Feng, W. W., Yang, W. H., 1973. On the contact problem of an inflated spherical nonlinear membrane. Journal of Applied Mechanics 40 (1), 209214.

Ho, C.-H., Henderson, K. A., Rorrer, G. L., 1995. Cell Damage and Oxygen Mass Transfer during cultivation of Nicotiana tabacum in a Stirred-Tank Bioreactor. Biotechnology Progress 11 (2), 140-145.

Kozicki, J., Kozicka, J., 2011. Human friendly architectural design for a small martian base. Advances in space research 48, 1997-2204.

Liu, K. K., Williams, D. R., Briscoe, B. J., 1996. Compressive deformation of a single microcapsule. Physical Review E 54 (6), 6673-6680.

Middelberg, A. P. J., O’Neill, B. K., Bogle, I. D. L., 1992a. A new model for the disruption of Escherichia coli by high-pressure homogenization I. Model development and verification. Trans. Inst. Chem. Eng. Part C (Food Bioprod. Process.) 70, 205-212.

Middelberg, A. P. J., O’Neill, B. K., Bogle, I. D. L., Gully, N. J., Rogers, A. H., Thomas, C. J., 1992b. A new model for the disruption of Escherichia coli by high-pressure homogenization II. A correlation for the effective cell strength. Trans. Inst. Chem. Eng. Part C (Food Bioprod. Process.) 70, 213-218.

Mooney, M., 1940. A theory of large deformation. Journal of Applied Physics 11, 582-592.

Pfeffer, W., 1891. Zur kenntnis der plasmahaut und der vacuolen etc. Abh. d. Math.-Phys. Kl. d. Sächs. Akad. d. Wiss. 16, 185-344. 
Smith, A. E., Moxham, K. E., Middelberg, A. P. J., 1998. On uniquely determining cell-wall material properties with the compression experiment. Chemical Engineering Science 53 (23), 3913-3922.

U.S Department of Defense, 1999. Department of Defense Design Criteria Standard Human Engineering. Tech. Rep. MIL-STD-1472F, Department of Defense United States of America.

Vlès, F., 1926. Les tensions de surface et les déformations de l'oeuf d'oursin. Archives de physique biologique 4, 263-284.

Walter, J., 2011. The Engine Indicator. The Canadian Museum of Making.

Yang, W. H., Feng, W. W., 1970. On axisymmetrical deformations of nonlinear membranes. Journal of Applied Mechanics 37, 1002-1011.

Zhang, Z., Thomas, C. R., 1993. Micro-manipulation: A new approach to studying animal cell damage in bioreactors. The genetic Engineer and Biotechnologist 13, 19-29. 